\title{
Structure, composition chimique et retraits de maturation du bois chez les clones d'Eucalyptus
}

\author{
H Baillères 1, B Chanson 2, M Fournier 2, MT Tollier ${ }^{3}$, B Monties ${ }^{3}$
}

\author{
${ }^{1}$ CIRAD-Forêt, 45 bis, avenue de la Belle-Gabrielle, 94736 Nogent-sur-Marne cedex; \\ 2 UMR CO23 CNRS-INRA-université de Bordeaux I, laboratoire de rhéologie du bois \\ de Bordeaux, BP 10, 33610 Cestas-Gazinet; \\ 3 INRA-INAPG, laboratoire de chimie biologique, centre de Grignon (CBAI), \\ 78850 Thiverval-Grignon, France
}

(Reçu le 8 décembre 1993 ; accepté le 5 mai 1994)

\begin{abstract}
Résumé - Contrairement à beaucoup d'arbres feuillus, le bois de réaction des Eucalyptus n'est pas caractérisé par les fibres $\mathrm{G}$. II peut cependant être caractérisé par ses déformations de maturation. La mesure des déformations résiduelles longitudinales de maturation (DRLM) par relaxation de contraintes en périphérie des troncs a permis un classement continu et quantitatif d'échantillons de bois de clones d'Eucalyptus hybrides du Congo. Ce type de mesure permet d'identifier mécaniquement le bois de tension. Les relations entre structure et propriétés mécaniques de ces bois ont été étudiées par une analyse ultrastructurale et chimique des échantillons, en particulier par l'examen de la composition monomérique des lignines par thioacidolyse. Les résultats présentés ici montrent qu'il existe : i) une forte variabilité des DRLM avec des valeurs élevées, même sur nos arbres peu penchés et peu excentrés, et malgré l'absence de fibres $G$; ii) une corrélation négative entre le niveau de DRLM, le taux de lignines et l'angle des microfibrilles de cellulose ; iii) une corrélation positive entre le niveau de DRLM et le rapport des taux de monomères de lignines : unités syringyles / unités guaiacyles. La signification de ces corrélations est discutée (causalité mécanique directe ou corrélations plus complexes entre variables structurales).
\end{abstract}

bois de tension / contraintes de croissance / Eucalyptus / lignines / angle des microfibrilles

Summary - Wood structure, chemical composition and growth strains in Eucalyptus clones. Contrary to a lot of hardwoods, the tension wood of Eucalyptus species is not characterised by G-fibres. It can however be characterised by its growth strains. The measurement of residual growth strains at the stem surface, by relaxing stresses at the periphery of trunks, allowed a continuous and quantitative classification of wooden samples coming from clones of hybrids Eucalyptus. This kind of measurement allows a mechanical identification of tension wood. The connections between structure and mechanical properties of these different woods were studied by ultrastructural and chemical analysis of samples, especially by a quantitative investigation of the monomeric compound of lignins by thioacidolysis. The results presented in this study show: i) a large variation of the residual growth strains with high values, in spite of the weak eccentricity, the good verticality and the absence in G-fibre in our trees; ii) a negative correlation between 
the level of the residual growth strains, the lignin content and the microfibril angle of cellulose (S2 layer of fibre); and iii) a positive correlation between the level of the residual growth strains and the ratio of the syringyl units to the guaiacyl units in lignin monomeric units. The significance of these correlations is discussed (direct mechanical causality or more intricate correlations between structural variables).

tension wood / growth stress / Eucalyptus / lignin / microfibril angle

\section{INTRODUCTION}

\section{Déformations de maturation et contraintes de croissance}

Le bois tend à se déformer à la fin de sa différentiation, lors de la formation de la paroi secondaire (Archer, 1986 ; Fournier et Guitard, 1994). En particulier, le bois dit «normal» tend à se rétracter (d'environ $0,1 \%$ ) dans le sens longitudinal des fibres. Cette déformation, appelée «déformation de maturation", permet d'expliquer les efforts internes importants supportés par le bois des arbres sur pied, généralement appelés «contraintes de croissance", qui se libèrent lors des opérations de transformations (de l'abattage au conditionnement) avec des effets néfastes (fentes en bout des billons, voilement et ruptures des débits). En effet, ces déformations de maturation ne s'expriment que très faiblement dans l'arbre sur pied car, du fait de la lignification, le bois périphérique nouvellement formé adhère fortement au noyau central de bois ancien, très rigide. II s'ensuit des efforts internes qualifiés de précontraintes ou encore d'autocontraintes, qui s'expliquent mécaniquement par l'action de l'ancien bois qui empêche la déformation du bois récemment mis en place. C'est ainsi que, dans le sens des fibres et dans le bois normal, l'empêchement de la déformation longitudinale de maturation (DLM), qui est un retrait, se traduit par une tension périphérique de ce nouveau bois.

Deux conséquences s'ensuivent.

D'une part, puisque les DLM ne se sont pas produites dans l'arbre sur pied, il est possible de les estimer expérimentalement par la mesure des déformations consécutives à leur relaxation (obtenue en désolidarisant le bois périphérique du reste de l'arbre par perçage, rainurage, sciage...). Les principes mécaniques et techniques de cette estimation sont maintenant bien établis (Archer, 1986 ; Baillères et al, 1992 ; Baillères, 1993 ; Fournier et al, 1994). La déformation mesurée, appelée «déformation résiduelle longitudinale de maturation» (DRLM), peut être confondue dans la plupart des cas avec la DLM (Fournier et al, 1991).

D'autre part, les DLM sont une propriété intrinsèque du bois (et non une déformation provoquée par un effort externe tel que poids ou vent, qu'il faudrait expliquer conjointement par l'intensité de l'effort incident et sa répartition sur une surface porteuse de bois compte tenu des rigidités du matériau). II est donc logique de chercher à les expliquer par la structure et la composition chimique du bois formé. En particulier, leur variabilité risque d'être explicable par la variabilité des structures et des compositions chimiques, conséquences éventuelles de différenciation particulières.

\section{Différentes définitions du bois de tension}

Chez les angiospermes, le bois dit «de réaction" ou "de tension» est notamment connu pour être le siège de précontraintes périphériques de tension plus intenses, caractérisées par des DRLM négatives (retraits) plus élevées que dans le bois normal (Trénard et Guéneau, 1975 ; Archer, 1986 ; 
Fournier, 1989 ; Mariaux et Vitalis-brun, 1983 ; Okuyama, 1990, 1992, 1993). Ceci peut constituer au sein de la même espèce et du même arbre une définition mécanique du bois de tension comme étant du bois «à plus fort retrait de maturation». Par ailleurs, selon la définition de l'IAWA (1964), le bois de réaction est «un bois qui présente des caractères anatomiques plus ou moins distinctifs; il se forme typiquement dans des portions de tiges penchées ou courbées et dans les branches; le végétal s'efforce de reprendre une position normal en réagissant de la sorte.» I| apparaît donc ici 2 définitions nouvelles et complémentaires du bois de réaction, l'une structurale, fondée sur des critères anatomiques, l'autre fonctionnelle, fondée sur l'aptitude mécanique de ce bois à réorienter les axes.

La définition mécanique du bois de réaction, de tension chez les feuillus, permet de relier fonction et structure.

D'une part, lorsque du bois à plus fort retrait de maturation est formé sur une face de la tige (par exemple, la face supérieure de l'axe incliné), cette face plus tendue «tire» sur l'autre et fléchit donc la tige entière (en redressant par exemple l'axe incliné) (Sinott, 1952 ; Hejnowicz, 1967 ; Fournier, 1989). Le bois de tension mécanique permet de modifier et de réguler courbures et inclinaisons des axes et accomplit donc une fonction de réorientation à condition qu'il soit différencié dans un secteur angulaire particulier (Wilson et Archer, 1977). Se posent alors indépendamment de nombreuses questions relevant de la physiologie des régulations : comment l'axe perçoit-il son déséquilibre et comment la différenciation particulière est-elle déclenchée, en réponse à un stimulus externe (Boyd, 1977a) ou à une consigne interne d'équilibre (Wilson et Archer, 1977) ? Quel est le rôle des hormones végétales (Morey, 1973) ?

D'autre part, le bois de tension mécanique est caractérisé par une rhéologie par- ticulière : un retrait de maturation plus fort. Cette propriété macroscopique, comme toute propriété rhéologique, est liée à la structure du bois à des échelles inférieures. Il reste alors à comprendre cette liaison.

Chez les Angiospermes, le bois de tension est anatomiquement caractérisé par la présence de fibres où une partie ou la totalité de la paroi secondaire est presque exclusivement composée de cellulose cristalline. Celle-ci a alors un aspect gélatineux d'où la dénomination de «fibre G». D'un point de vue ultrastructural, l'angle des microfibrilles (AMF), c'est-à-dire l'inclinaison moyenne des microfibrilles de cellulose par rapport à l'axe de la fibre, est très faible dans la couche $G$. Biochimiquement, le bois de tension est moins lignifié. La présence de fibre $G$ est habituellement le marqueur anatomique le plus facile à mettre en évidence. Cependant, dans le cas d'Eucalyptus regnans, Chafe (1977) a effectivement mis en évidence la présence de fibres peu lignifiées sans couche $G$ caractéristique. Par la suite Fisher et Wassmer Stevenson (1981) ont montré que ce résultat n'est pas exceptionnel puisque, sur un échantillon de 122 espèces, seules $46 \%$ de celles-ci possèdent un bois de tension à couche $\mathrm{G}$ caractéristique.

\section{Structure des lignines et bois de tension}

Les lignines constituent la fraction phénolique la plus importante des parois végétales. Ce sont des polymères tridimensionnels dont les 3 principaux monomères constitutifs sont l'alcool coumarylique, l'alcool coniférylique et l'alcool sinapylique, caractérisés respectivement par les cycles de type H, G ou S (fig 1).

Le type de liaison intermonomérique le plus fréquemment rencontré dans les lignines est la liaison $\beta-0-4$ alkyl aryl ether (fig 1). D'autres types de liaisons intermonomériques font intervenir des liaisons carbone-carbone 


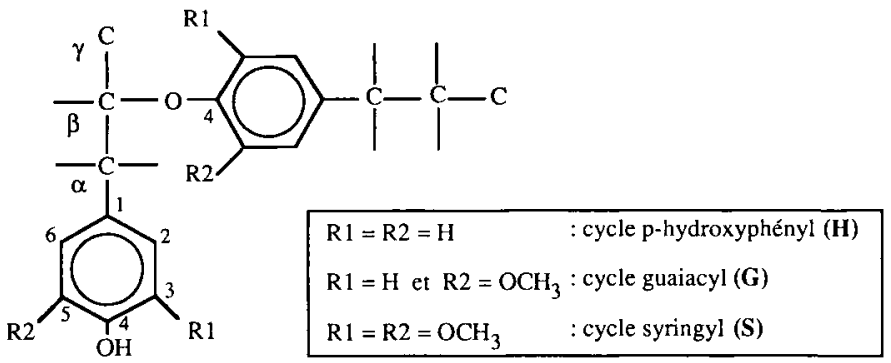

Fig 1. Structure et nomenclature $(H, G$ et S) des unités phénylpropanes des monomères constitutifs des lignines. Structure de la liaison aryl alkyl ether $(\beta-0-4)$ la plus fréquente. par exemple. La thioacidolyse, utilisée dans ce travail, coupe de façon spécifique les liaisons $\beta-O-4$ et permet de caractériser la composition monomérique des lignines (Monties, 1991 ; Rolando et al, 1992).

La teneur relative des monomères $G$ et $S$ a une valeur chimiotaxinomique : les conifères sont caractérisés par des lignines presque exclusivement constituées d'unités Guaiacyles alors que les angiospermes contiennent en part approximativement égales les 2 types $\mathrm{G}$ et $\mathrm{S}$. Elle a aussi une valeur xylologique, notamment dans le cas qui nous intéresse ici du bois de tension. À l'exception du bois de réaction des Conifères (bois de compression), l'analyse des bois ne révèle qu'une très faible proportion d'unités H (Lewin et Goldstein, 1991).

La structure des lignines dans le bois de tension a été rarement étudiée. Morohoshi et Sakakibara (1971) ont montré par oxydation au nitrobenzène que le rapport S/G n'augmentait que faiblement dans le bois de tension de Fraxinus mandschurica, ils ont conclu que la composition des lignines du bois de tension est peu différente de celle du bois opposé. Des résultats similaires ont été obtenus pour le bois de tension de Betula papyrifera et d'Arbutus menziesii (Starkanen et Hergett, 1971). Cependant, Bland (1958 et 1961), par oxydation au nitrobenzène sur Eucalyptus goniocalyx, a rapporté que le rapport $S / G$ est significativement plus faible dans le bois opposé. II a remarqué également, chez cette espèce, que la lignine du bois de tension est plus fermement liée à l'ossature polysaccharidique. Par ailleurs, Lapierre et al (1986a) ont observé une légère diminution du rapport $\mathrm{S} / \mathrm{G}$ mais qui ne semble pas significative dans le bois de tension de Populus euramericana cv 1214 ; ces résultats de thioacidolyse ne peuvent être comparés directement à l'oxydation au nitrobenzène. En revanche, notons que, dans toutes ces études, le bois de tension est défini par des critères morphologiques fondés sur la définition fonctionnelle (échantillons prélevés sur la face supérieure de tiges inclinées et excentrées), voire anatomiques (présence de fibres $\mathrm{G}$ ).

\section{Interprétation des relations observées entre structure du bois et déformations de maturation}

La structure anatomique et biochimique du bois de tension étant décrite, il faut alors se pencher sur l'interprétation physique des relations entre structure du bois et DLM. Des modélisations micro-mécaniques permettent d'exprimer la DLM à l'échelle de la paroi cellulaire en fonction de 2 phénomènes supposés moteurs, le gonflement de la matrice amorphe caractéristique de la lignification et le retrait des microfibrilles, caractéristique de la cristallisation de la cellulose (fig 2). Une querelle oppose Boyd (1972, 1977a et b, 1985), qui nie l'existence du second phénomène, à Bamber (1987) qui le tient au contraire pour prépondérant. Les modèles (Boyd, 1972 ; Archer, 1987 ; 
Archer, 1988 ; Yamamoto et Okuyama, 1988 ; Bonnin et Demanet, 1993) intègrent des paramètres tels que l'inclinaison des microfibrilles par rapport à l'axe de la cellule dite "angle des microfibrilles» (AMF), l'épaisseur de la couche $G$, les teneurs volumiques en microfibrilles et en matrice de lignines et d'hémicelluloses, et les caractéristiques élastiques des microfibrilles et de la matrice in situ. Ces caractéristiques rhéologiques, difficiles à mesurer directement, sont vraisemblablement dépendantes de la structure biochimique des composants et de leur organisation dans l'espace. Ces modèles, confortés par l'expérience, s'accordent tous sur l'importance de l'angle des microfibrilles (plus I'AMF est fermé, plus la DLM est un retrait élevé ; la DLM devient un gonflement pour les AMF très ouverts). Ils sont un support de réflexion qui permet d'identifier des caractéristiques pertinentes pour l'interprétation de la variabilité des DLM en mettant en évidence les paramètres qui interviennent dans le problème physique. Mais ils ne sont pas encore un outil de prédiction quantitatif car, d'une part, leur réalisme est discutable (la querelle entre Bamber et Boyd n'est pas résolue, la prise en compte des cinétiques de lignification, des liaisons entre cellules... n'est pas réalisée) ; d'autre part, les paramètres qui interviennent sont parfois difficilement mesurables (rhéologie des constituants pariétaux). Enfin, ils ne varient. généralement pas de façon indépendante sur les échantillons observés (un AMF élevé correspond généralement à une forte teneur en lignines, la cause physique ou biologique de ce phénomène n'est pas immédiate et de toute façon pas modélisée).

Il faut noter que, chez les Gymnospermes, il existe aussi un bois de réaction

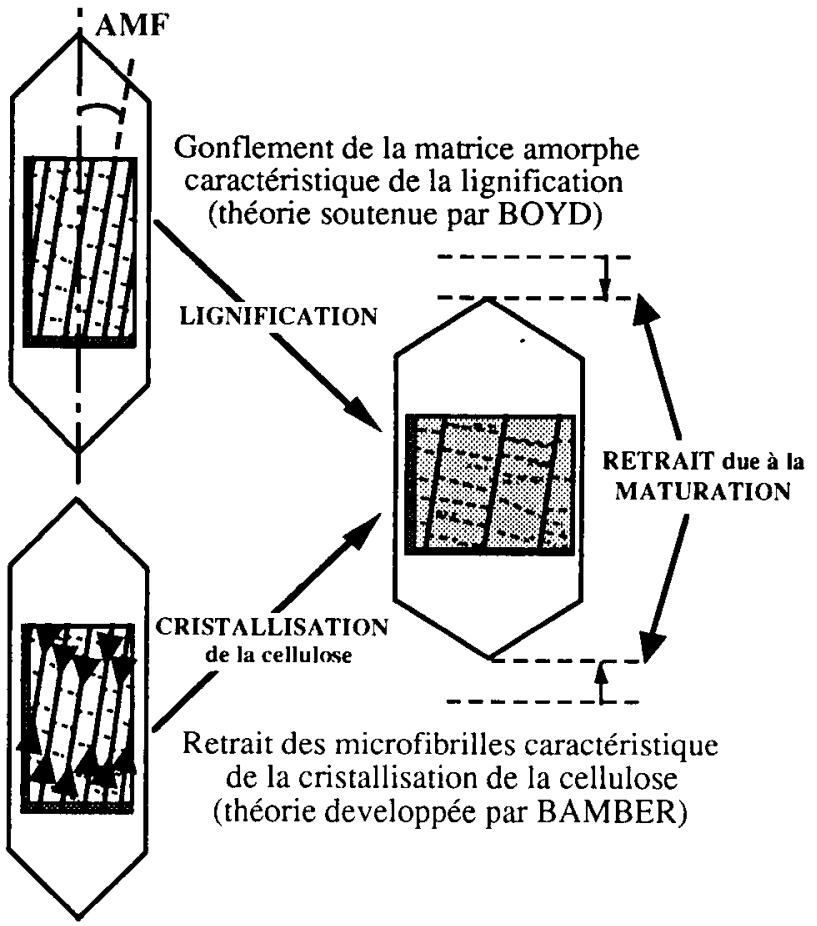

Fig 2. Schéma synthétique des 2 origines probables de la déformation longitudinal de maturation à l'échelle de la paroi cellulaire. 
fonctionnel, le bois de compression, qui est formé, à l'inverse, dans la partie inférieure des tiges inclinées. Ce bois peut également être défini par sa structure : trachéides arrondies avec d'importants méats inter-cellulaires, bois fortement lignifié, AMF très ouvert $\left(>40^{\circ}\right)$, épaississements spiralés sur la face externe de la couche S2 (Low, 1964), augmentation très significative de la concentration en lignines et de la proportion d'unités $\mathrm{H}$ (Lapierre et al, 1987 ; Rolando et al, 1992). Enfin, il est aussi caractérisable mécaniquement : dans le bois de compression la DLM est un gonflement (au lieu d'un retrait dans le bois normal ou opposé ; Archer, 1986; Fournier, 1989), explicable par la valeur élevée de I'AMF. Ce bois accomplit donc sa fonction de réorientation par un mécanisme inverse de celui du bois de tension en «poussant» la face opposée.

Le présent travail a pour objet d'analyser les relations entre valeurs de DLM, en mettant à profit l'importante variabilité constatée entre des bois "très" et "faiblement» tendus, et certaines propriétés ultrastructurales et biochimiques du bois de clones d'Eucalyptus plantés au Congo. Les objectifs sont, après un choix d'échantillons sur la base des DRLM (donc à partir d'une définition mécanique et non anatomique ou fonctionnelle des bois de tension), de quantifier le continuum des structures entre bois normal et bois de tension, de mettre en évidence des caractéristiques spécifiques en l'absence de fibres $\mathrm{G}$, en évitant de se limiter à des bois de tension très typiques situés sur la face supérieure d'axes très inclinés.

\section{MATÉRIEL ET MÉTHODES}

\section{Matériel}

Les échantillons proviennent de 10 arbres choisis parmi 4 clones d'Eucalyptus hybrides (UAIC : 1 $45,2-3$, L2-131, 18-14) utilisés en plantations industrielles ou expérimentales au Congo. Les espèces à l'origine des hybrides sont incertaines (Vigneron, 1992).

Les mesures de DRLM (à raison d'une couronne de 4 à 8 mesures et prélèvements par arbres) ont permis de classer les échantillons de bois analysés en fonction des valeurs obtenues (exprimées en microdéformation $=1 \mu \mathrm{m} / \mathrm{m}$ ). Ce tri a été réalisé sans distinction d'âge ni de clone.

Pour le dosage et la caractérisation des lignines, 55 échantillons ont été répartis en 9 classes comprenant chacune 4 à 8 échantillons (tableau I). Pour chaque classe, la moyenne des DRLM a été calculée, pondérée par rapport à la masse respective de chaque prélèvement (environ $1,5 \mathrm{~g}$ ) réalisé exactement au niveau de la mesure mécanique.

Pour la réalisation de coupes anatomiques (coupes transversales colorées et coupes tangentielles pour la mesure de l'angle de microfibrilles), nous avons utilisé des échantillons prélevés dans chaque classe.

\section{Méthodes}

\section{Mesure des DRLM}

La méthode des 2 rainures réalisée sur une petite base de mesure libère entièrement la contrainte longitudinale de sorte que la déformation mesurée est bien conforme à la définition de la DRLM donnée en introduction. En pratique, cette méthode utilise un extensomètre à jauges résistives (capteur de déformation HBM type DD1) fixé parallèlement au fil du bois par l'intermédiaire de pointes de compas enfoncées sur une profondeur de 1 à $2 \mathrm{~mm}$ à la surface de l'arbre écorcé. Ce capteur est relié à une centrale d'acquisition portable (Captels, Système Alco). La déformation longitudinale obtenue, sur une base de mesure de $14 \mathrm{~mm}$, résulte de l'usinage par sciage manuel de 2 entailles perpendiculaires au fil du bois, situées de part et d'autre du capteur de déformation, à $5 \mathrm{~mm}$ de chaque pointe, et profondes d'environ $7 \mathrm{~mm}$ (Baillères et al, 1992 ; Baillères, 1994 ; Fournier et al, 1994).

\section{Étude anatomique}

Des coupes de bois ont été réalisées sur une cinquantaine d'échantillons (conservés dans l'alcool à $70^{\circ}$ ) couvrant toutes les classes de DRLM 
Tableau I. Teneurs et composition monomérique des lignines en unités de type G (Guaiacyle) et S (Syringyle).

\begin{tabular}{|c|c|c|c|c|c|c|}
\hline $\begin{array}{c}\text { Classes de } \\
\text { DRLM en } \\
\text { microdéformations a }\end{array}$ & $\begin{array}{c}\text { Moyenne } \\
\text { pondérée } \\
\text { des DRLM }\end{array}$ & $\begin{array}{c}\text { Lignine de } \\
\text { Klason }{ }^{\circ}\end{array}$ & $S c$ & $G^{\mathrm{c}}$ & $S+G^{c}$ & $S / G$ \\
\hline $420-700$ & 507 & $32,1 \pm 0,13$ & $1232 \pm 45$ & $834 \pm 6$ & $2066 \pm 51$ & 1,47 \\
\hline $700-980$ & 888 & $29,8 \pm 0,2$ & $1405 \pm 35$ & $904 \pm 9$ & $2309 \pm 44$ & 1,55 \\
\hline $980-1260$ & 1133 & $29,5 \pm 0,04$ & $1282 \pm 103$ & $826 \pm 61$ & $2108 \pm 165$ & 1,55 \\
\hline $1260-1540$ & 1390 & $29,2 \pm 0,06$ & $1460 \pm 60$ & $892 \pm 33$ & $2352 \pm 93$ & 1,64 \\
\hline $1540-1820$ & 1679 & $29,2 \pm 0,11$ & $1432 \pm 46$ & $806 \pm 20$ & $2238 \pm 66$ & 1,78 \\
\hline $1820-2100$ & 1981 & $28,5 \pm 0,4$ & $1273 \pm 6$ & $827 \pm 4$ & $2100 \pm 11$ & 1,54 \\
\hline $1820-2100$ & 2030 & $27,7 \pm 0,1$ & $1349 \pm 129$ & $793 \pm 75,6$ & $2142 \pm 210$ & 1,70 \\
\hline $2100-2380$ & 2276 & $26,9 \pm 0,06$ & $1404 \pm 6$ & $773 \pm 8$ & $2177 \pm 14$ & 1,82 \\
\hline $2380-2940$ & 2761 & $25,6 \pm 0,1$ & $1372 \pm 20$ & $734 \pm 12$ & $2009 \pm 31$ & 1,87 \\
\hline 2940 et + & 3363 & $25,0 \pm 0,02$ & $1354 \pm 1$ & $699 \pm 23$ & $2053 \pm 22$ & 1,96 \\
\hline
\end{tabular}

Pour les teneurs en lignines de Klason et en monomères, le premier chiffre est la valeur moyenne, le second est l'écart type. La classe $[1820 ; 2100]$ comporte 2 échantillons différents. a En valeur absolue ; ${ }^{b}$ exprimés en pourcentage de la masse du résidu pariétal ; ${ }^{c}$ exprimés en micromoles par gramme de lignine de Klason.

sur un microtome JUNG 240. Les coupes transversales, d'une épaisseur nominale de $16 \mu \mathrm{m}$ ont été colorées par la double coloration bleu astra/safranine pour mettre en évidence les couches $G$ des fibres de bois de tension (Dot et Gautié, 1928). L'ensemble des coupes et colorations testées ont également servi pour la description anatomique des différents types de bois mis en évidence (variation du plan ligneux, forme des cellules, épaisseur des parois, etc).

\section{Mesure de l'angle de microfibrilles (AMF)}

La mise en évidence de l'angle des microfibrilles a été réalisée à partir de coupes tangentielles. Elles subissent une déshydratation totale par passage à l'étuve sous vide à $105^{\circ} \mathrm{C}$ puis sont réhydratées. Ce traitement, réalisé 3 fois par coupe, permet de provoquer l'ouverture de microfissures dans la paroi secondaire, en particulier au niveau des ponctuations des fibres. La direction de ces microfissures suit l'orientation des fibrilles de cellulose. Á la suite de ce traitement, les coupes ont été contrastées par coloration au Paragon ${ }^{\oplus}$. Après avoir constaté la faible variabilité radiale de l'AMF dans le bois correspondant à une zone de mesure mécanique, liée à l'absence de cernes distincts, nous avons pu limiter le nombre de mesures. La mesure de l'angle des microfibrilles a été exécutée sur 5 à 6 coupes à raison d'une dizaine de fibres par coupe sur un microscope optique équipé d'un goniomètre.

Cette méthode a été validée sur un nombre réduit d'échantillons couvrant toute la gamme de DRLM par observation directe au microscope électronique, selon la technique décrite par Mariaux et Vitalis-Brun (1983).

\section{Analyse des lignines}

\section{Observations histochimiques}

À partir de coupes transversales réalisées dans le même échantillon, nous avons utilisé la réaction de Wiesner (Dot et Gautié, 1928) pour mettre en évidence des variations de coloration de lignines selon le protocole suivant :

-i) nettoyage des coupes par traitement à l'hypochlorite de sodium $(20 \%)$ et acide acétique $\left(25^{\circ}\right)$ puis rinçage à l'eau distillée (des essais préliminaires ont permis de vérifier que ce traitement ne modifie pas significativement l'intensité de la coloration) ;

- ii) déshydratation ménagée à l'alcool éthylique ;

- iii) imprégnation (10 $\mathrm{min}$ ) au phloroglucinol ( $2 \%$ phloroglucine dans alcool éthylique $100^{\circ}$ ); 
- iv) révélation à l'acide chlorhydrique $\left(37^{\circ}\right)$;

- v) rinçage rapide à l'eau distillée et montage aqueux entre lame et lamelle.

La réaction de Wiesner révèle les fonctions aldéhyde des unités $S$ et $G$ (coniféraldéhyde, aldéhydes cinnamiques et les benzaldéhydes), en donnant un chromogène de couleur rouge qui permet de classer les coupes provenant des différents bois en fonction de l'intensité de la couleur qui traduit une plus ou moins grande quantité de lignines (Clifford, 1974 ; Geiger et Fuggerer, 1979 ; Yoshizawa et al, 1993). La coloration obtenue est fugace et les observations doivent être menées impérativement dans les 20 min qui suivent le début de la réaction.

\section{Préparation des résidus pariétaux}

Les résidus pariétaux sont obtenus en passant la totalité des échantillons au broyeur Wiley ${ }^{(i)}$ muni d'un tamis de 40 mesh $(0,4 \mathrm{~mm})$ et ensuite par extraction au Soxhlet en utilisant la séquence de solvant toluène-éthanol 2:1 -> éthanol $\rightarrow>$ eau, qui permet d'éliminer les phénols solubles et les autres composés "extractibles», non liés aux parois. Les résidus sont ensuite amenés à l'état anhydre.

\section{Détermination de la lignine de Klason}

La teneur en lignines est mesurée selon le protocole gravimétrique de Klason sans pré-hydrolyse acide d'après Effland (1977). Cette technique présente 2 phases :

i) $\mathrm{H}_{2} \mathrm{SO}_{4} 72 \%$ pendant $2 \mathrm{~h}$ à $20^{\circ} \mathrm{C}$;

ii) chauffage sous reflux pendant $3 \mathrm{~h}$ après dilution de l'acide $0,5 \mathrm{~N}$.

Le résidu insoluble (exprimé en \% du résidu pariétal) obtenu après filtration, lavage et séchage correspond à la lignine de Klason après correction de la teneur en cendres (incinération à $550^{\circ} \mathrm{C}$ ).

\section{Composition monomérique de la lignine}

Les lignines sont caractérisées par thioacidolyse. Cette technique de dépolymérisation des lignines, par rupture de la liaison $\beta-0-4$ alkyl aryl ether (fig 1 ), permet d'isoler uniquement les monomères $S$ et $\mathrm{G}$ des unités «non condensées» (Monties, 1991 ; Rolando et al, 1992). Les teneurs en unités $S$ et $G$ ainsi obtenues ne représentent donc pas la fréquence absolue de ces monomères dans les lignines étudiées mais seulement la fréquence des liaisons $\beta-0-4$ alkyl aryl ether dans lesquelles ces unités sont impliquées. II faut noter que, dans le bois, cette méthode permet de caractériser sans équivoque la plus grande partie des lignines (Rolando et al, 1992).

La thioacidolyse consiste en une solvolyse de $15 \mathrm{mg}$ de résidu pariétal dans un mélange dioxane/éthanéthiol $(9: 1, v / v)$ contenant $0,2 \mathrm{~N}$ d'éthérate de bore trifluoré, durant $4 \mathrm{~h}$ dans un bain d'huile à $100^{\circ} \mathrm{C}$ (Lapierre et al, $1986 \mathrm{~b}$; Rolando et al, 1992).

Les produits de thioacidolyse monomériques $S$ et $G$ sont récupérés par extraction au dichlorométhane puis séparés et quantifiés par chromatographie en phase gazeuse.

\section{RÉSULTATS}

\section{Valeurs des DRLM mesurées}

Toutes les DRLM évaluées dans cette étude sont des retraits, ce sont donc des valeurs négatives. Dans la suite de cet article, par mesure de simplification, nous les exprimons en valeur absolue. Elles s'échelonnent entre 400 et 3500 microdéformations $(=1 \mu \mathrm{m} / \mathrm{m})$. Les valeurs les plus fréquentes sont situées entre 800 et 1400 microdéformations (Baillères, 1994 ; Fournier et al, 1994).

\section{Recherche de bois de tension et caractéristiques anatomiques}

La double coloration bleu astra/safranine n'a pas permis de distinguer des fibres à paroi $G$ typique. En effet, à cause des variations d'affinité de la paroi cellulaire, ces colorants ne peuvent pas toujours être utilisés comme des indicateurs fiables. Les Eucalyptus hybrides étudiés sont donc, de ce point de vue, comparables à Eucalyptus regnans (Chafe, 1977).

\section{Coloration des lignines sur coupes transversales}

La coloration de Wiesner permet de révéler une évolution continue de la coloration attri- 
buable à la présence de fonctions cinnamaldéhydes et donc à la lignification. Dans la paroi S2, elle est peu marquée dans les zones à fortes valeurs de DRLM mais suffisante pour indiquer la présence de lignines. Elle est intense dans les zones à faibles valeurs de DRLM. Les valeurs intermédiaires sont caractérisées soit par une coloration intermédiaire soit par la succession de zones fortement et faiblement colorées.

Le bois correspondant aux faibles valeurs de DRLM présente des fibres au contour arrondi et une plus forte proportion de parenchyme diffus. Le groupe de parois $\mathrm{ML}+\mathrm{P}+$ $\mathrm{S} 1$ est plus épais et plus coloré par la réaction de Wiesner.

À l'opposé, dans le bois à fortes DRLM, les fibres sont bien alignées et possèdent un contour rectangulaires. Le groupe de parois $M L+P+S 1$ est moins épais et moins coloré par la réaction de Wiesner.

\section{Variation du taux de lignine de Klason (fig 3)}

La technique utilisée permet d'obtenir la teneur totale de lignines (par gravimétrie) avec le risque de contaminations par la présence de polyosides hémicellulosiques et de protéines. Cette teneur varie de 25 à $32 \%$ du résidu pariétal. La régression linéaire DRLM en fonction du taux de lignine de Klason est négative, $R=-0,97$ significatif au seuil de $1 \%$ (fig 3 ). Ce résultat concorde avec celui obtenu sur coupes minces par la réaction de Wiesner, indiquant une bonne corrélation entre teneur en lignine de Klason et teneur en fonction cinnamaldéhyde.

\section{Variations des taux des monomères $S$ et $G$ et du rapport $S / G$ (fig 4)}

Le taux d'unités $\mathrm{G}$ diminue sensiblement lorsque le niveau de DRLM augment alors qu'il est difficile de se prononcer sur l'évolution des taux d'unités $S$ (fig 4a). La conséquence est donc une diminution du taux d'unités non condensées $(S+G)$. Ce résultat reste à confirmer par une analyse sur un plus grand nombre d'échantillons. On observe une légère discontinuité aux environs de 1500 microdéformations : la diminution du taux d'unités $G$ avec la DRLM paraît s'accentuer pour des DRLM supérieures à 1500 microdéformations, alors que le taux d'unités $S$ semble, lui, croître entre 0 et 1500 microdéformations. La dispersion des valeurs est cependant forte, ce

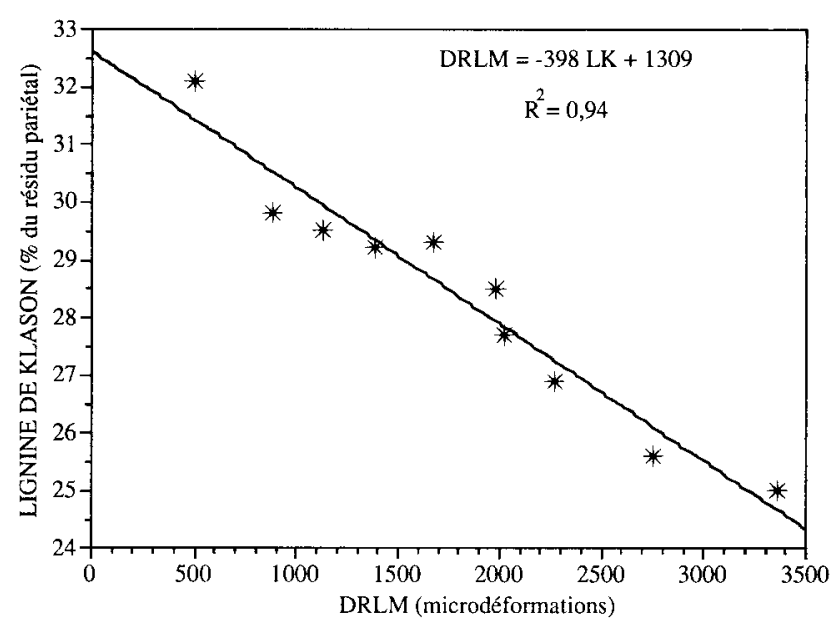

Fig 3. Taux de lignine de Klason en fonction des déformations longitudinales résiduelles de maturation (DRLM). 
qui rend délicate une interprétation plus approfondie de ces tendances.

Le rapport $S / G$ varie continûment de 1,47 à 1,96 (fig 4b). Le coefficient de corrélation linéaire entre la DRLM et S/G est positif et égal à 0,89 , significatif au seuil de $1 \%$.

\section{Variation de l'angle de microfibrilles de cellulose de la paroi S2}

Cet angle varie de 0 à $27^{\circ}$ avec une valeur moyenne de $9,5^{\circ}$. L'ajustement d'un polynôme de degré 3 à l'ensemble des points
(DRLM, AMF) est motivé par le changement de signe de la DRLM généralement observé à partir d'un angle d'environ $30^{\circ}$ (fig 5), elle devient alors un gonflement. Le coefficient de corrélation non linéaire $(r=0,97)$ est significatif au seuil de $1 \%$.

\section{DISCUSSION}

Les relations décrites ici entre DRLM et angle de microfibrilles ou lignines de Klason sont analogues aux tendances mises en évidence par Boyd (1977b et 1980), au
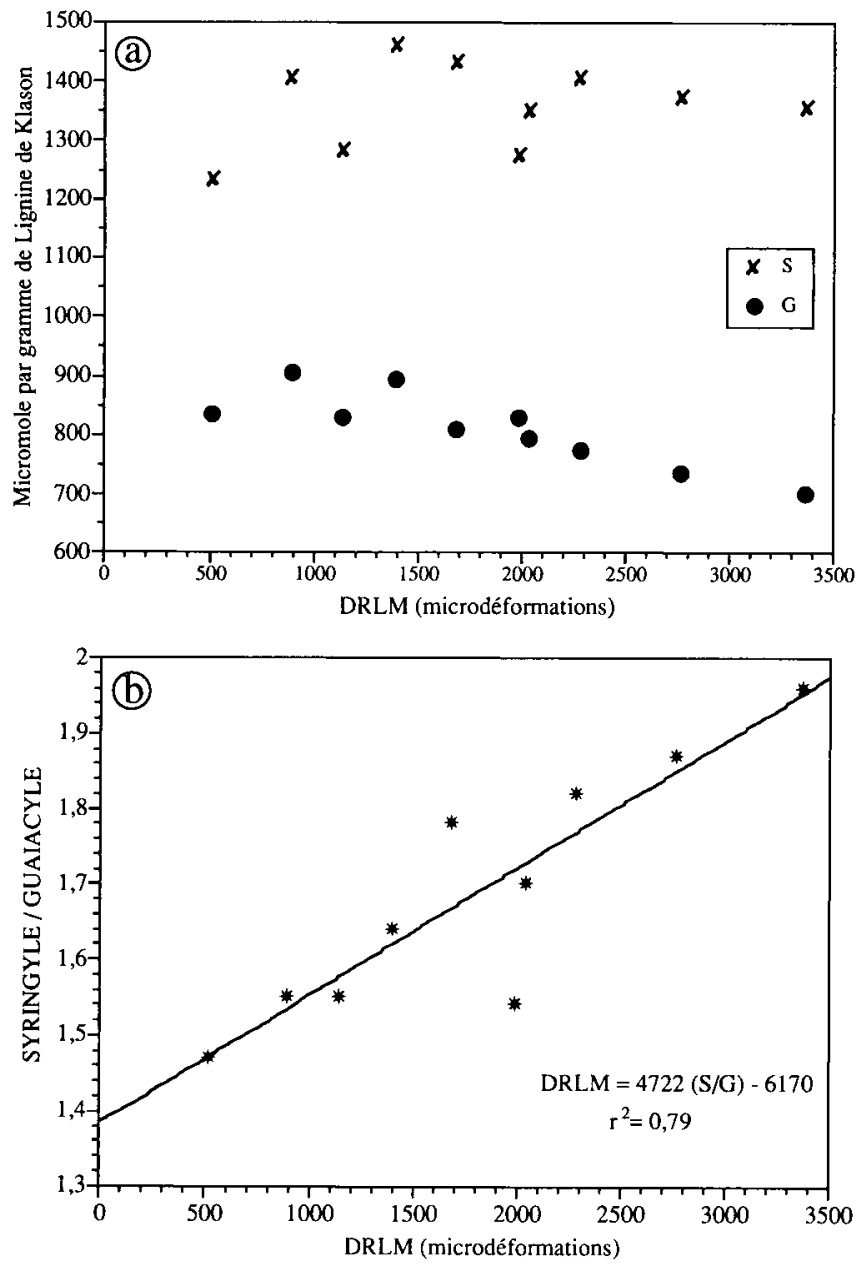

Fig 4
Fig 4. Variations des taux d'unités monomériques non condensées $S$ et $G$ ainsi que du rapport $S / G$ en fonction du niveau de DRLM. 
niveau intra-arbre sur 17 Eucalyptus regnans et un Eucalyptus sieberi inclinés de forêt naturelle, tous âgés de 40 ans, avec de 5 à 20 mesures par couronne. Cet auteur montre des valeurs de contraintes de croissance variant en moyenne de 1 à 5 (de 1 à 3 chez les arbres "normaux", de 1 à 20 chez les arbres en cours de réorientation). Bien que la technique de mesure des déformations soit différente et que les résultats de Boyd soient exprimés en contrainte et non en déformation, il est intéressant de noter que ce rapport de 1 à 5 est comparable à celui de 1 à 7 que nous avons obtenu sur de jeunes clones de plantation très peu inclinés. L'absence d'inclinaison significative n'est donc pas un critère fiable d'absence de bois de réaction. Ce résultat, conforté par de nombreuses observations sur différentes espèces (Archer, 1986 ; Fisher et Wassner Stevenson, 1982 ; Fournier et al, 1944), ne contredit pas l'interprétation fonctionnelle du bois de tension : un axe vertical peut se réorienter pour chercher la lumière ; de plus, la verticalité observée peut être le résultat d'une réorientation efficace ayant demandé la formation de bois de tension.

L'absence de bois de tension à fibres $G$ n'est pas surprenante, ils confirment les résultats de Chafe (1977) et de Nicholson et al (1975) qui ont montré que la présence de fibres $\mathrm{G}$ caractéristiques est rare dans le genre Eucalyptus. De même les bois à très forte DRLM restent faiblement lignifiés, ce qui s'accorde avec les résultats de Scurfield (1972).

Concernant les variations des angles de microfibrilles, nos résultats concordent avec les observations de Yoshida et al (1992) sur Eucalyptus rubida, Okuyama et al (1990, 1992) sur Tulipifera liriodendron et Magnolia obovata (espèces qui ne possèdent pas de couche G). En revanche Boyd (1980) donne des valeurs beaucoup moins dispersées, de 6 à $11^{\circ}$, mais avec une moyenne comparable. Ces résultats sont en accord avec les modélisations micro-mécaniques : lorsque I'AMF diminue, par rapport à l'axe de la cellule, d'une part le gonflement de la matrice se manifeste moins et d'autre part le retrait des microfibrilles s'exprime davantage ; il s'ensuit une augmentation du retrait de maturation axial (fig 6).

La diminution de la DRLM lorsque la teneur en lignines de Klason augmente concorde avec les résultats de Boyd (1977b) sur Eucalyptus regnans et d'Okuyama et al (1992, 1993) sur Liriodendron tulipifera, une espèce qui ne possède pas de bois de tension à fibres $\mathrm{G}$. Ce résultat semble en accord avec les modèles micro-mécaniques qui prévoient qu'à angle de microfibrilles fixé

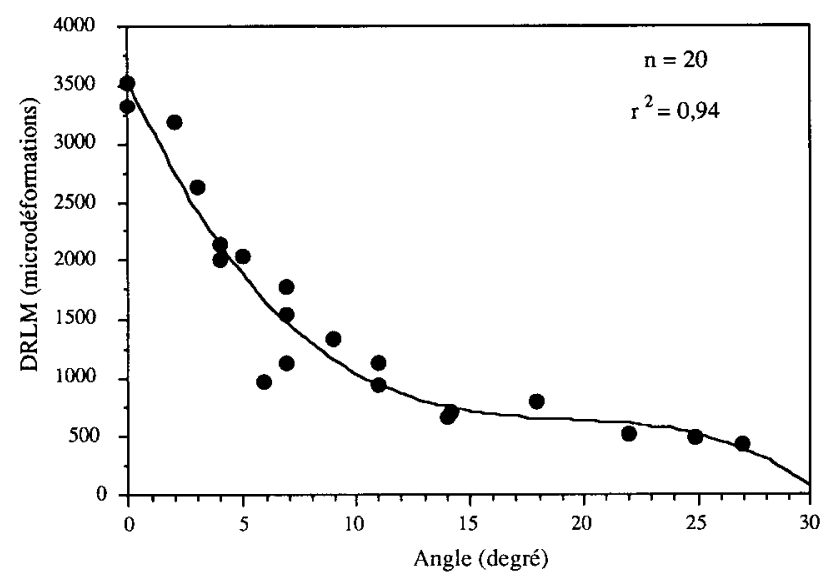

Fig 5. Variation de l'angle de microfibrilles de la paroi S2 des fibres en fonction du niveau de DRLM. 


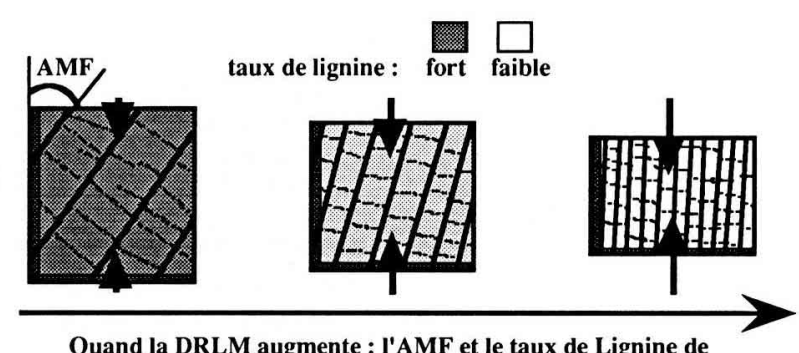

Quand la DRLM augmente : I'AMF et le taux de Lignine de Klason décroissent conjointement
Fig 6. Représentation schématique des observations mécaniques et histologiques. le retrait de maturation diminue avec la teneur en matrice. D'après les résultats de Schwerin (1958), on peut admettre qu'une augmentation de la teneur en lignines de Klason traduit une augmentation de la proportion de matrice (lignines et hémicelluloses). Cette augmentation est conjointe à une diminution de la teneur (complémentaire) en cellulose cristalline (Okuyama et al, 1992, 1993). Toutefois, dans la mesure où, dans notre échantillon, l'angle des microfibrilles augmente significativement en même temps que la teneur en lignines, il est impossible de discriminer celle des 2 variables qui explique physiquement la variation de DRLM. Dans le même ordre d'idées, d'autres auteurs ont montré que le bois de tension du hêtre (anatomiquement défini par la quantité de fibres G) et de l'Eucalyptus était caractérisé par une augmentation de la teneur en cellulose (Ruel et Barnoud, 1978 sur Fagus sylvatica; Schwerin, 1958 sur Eucalyptus goniocalyx). Par ailleurs, des résultats récents mettent en évidence une augmentation significative de la DRLM avec la teneur en $\alpha$-cellulose et avec le taux de cristallinité de la cellulose (Okuyama et al, 1990, 1992 chez Tulipifera liriodendron, essence feuillue sans couche $\mathrm{G}$ ).

Remarquons alors que, par son AMF et son taux de lignines élevés, le bois à faible DRLM est structuralement proche du bois de compression des gymnospermes.

Le bois à forte DRLM présente la structure habituellement décrite du bois de ten- sion pour les espèces feuillues sans couche G. La définition mécanique semble concorder avec la définition structurale. Notons qu'elle concorde également avec la définition fonctionnelle dans la mesure où nous avons constaté, en analysant l'hétérogénéité des DRLM sur la circonférence des arbres mesurés, qu'une forte DRLM sur une face correspond à une faible DRLM sur la face opposée.

Sur coupes tranversales, les localisations des zones à teneurs en lignines différentes montrent assez fréquemment une succession de couches de bois plus ou moins lignifiées. Si l'on admet, à partir des résultats précédents, que la teneur en lignines caractérise la DRLM, cela signifie que des gradients radiaux importants de DRLM ne sont pas rares. II s'agit alors d'une répartition diffuse et complexe de bois de réaction déjà observée sur des hêtres droits et verticaux (Lenz et Strassler, 1959). Delavault (1991) a montré sur de jeunes Eperua falcata que de telles répartitions ne sont pas aléatoires mais interprétables par les variations fréquentes d'inclinaison et de forme de jeunes arbres ; elles traduisent alors une recherche assez fine de la position d'équilibre de l'arbre. En l'absence d'une localisation précise du bois de tension sur des rondelles entières, à plusieurs niveaux dans l'arbre, ceci n'est cependant pas démontré sur nos Eucalyptus. On retiendra toutefois que, sur des arbres peu penchés, l'inclinaison ne permet pas de localiser le bois de tension et qu'en outre ce bois 
n'a aucune raison d'être situé sur un secteur angulaire unique allant de la moelle à la périphérie.

Le rapport moyen $S / G(1,6)$ est comparable à celui cité par Rolando et al (1992), obtenu par thioacidolyse sur Populus euramericana cv 1214.

De plus, l'évolution de ce rapport avec les DRLM est significative au seuil de $1 \%$. Elle n'est pas conforme aux résultats de Morohoshi et Sakakibara (1971) sur Fraxinus mandschurica obtenus par une méthode différente (oxydation au nitrobenzène) et opposée aux résultats cités par Rolando et al (1992) sur Populus euramericana cv 1214 ( $\mathrm{S} / \mathrm{G}=1,55$ dans le bois opposé et $\mathrm{S} / \mathrm{G}=$ 1,48 dans le bois de tension). En revanche, elle s'accorde avec les résultats de Bland (1958 et 1961), obtenus par oxydation au nitrobenzène sur Eucalyptus goniocalyx.

La caractérisation mécanique du bois de tension utilisée ici, en permettant une quantification précise et continue, favorise certainement l'obtention de résultats significatifs. De fait, la caractérisation anatomique ou morphologique des bois de tension usuellement réalisée ne permet pas de distinguer des bois de tension intenses ou faibles. De plus, le bois diamétralement opposé à une zone de bois de tension ne correspond pas forcément à du bois peu tendu (Chanson, 1993). Ainsi, le bois opposé analysé par Rolando et al (1992) avait été défini comme étant le secteur diamétralement opposé au secteur de bois de tension situé sur la face supérieure d'un arbre incliné. Nos résultats montrent que ce bois opposé aurait pu avoir un niveau de DRLM relativement proche de celui du bois de tension caractéristique. Mais, surtout, il est très vraisemblable que ces résultats sont dus à la différence ultrastructurale essentielle entre le bois de tension du peuplier ou du frêne (caractérisé par la présence de fibres $\mathrm{G}$ ) et le bois de tension des Eucalyptus étudiés.

La question est enfin de savoir si la relation entre la DRLM et le rapport S/G s'ex- plique par une causalité mécanique directe. Par exemple, des variations de rapport S/G pourraient traduire des différences de gonflement ou de rigidité de la matrice de lignines et d'hémicelluloses, paramètres qui influencent directement la DLM (cf les modèles micro-mécaniques décrits en introduction). Dans le même ordre d'idées, d'autres auteurs ont montré que les hémicelluloses avaient une composition et une teneur (diminution) significativement différente dans le bois de tension (anatomiquement défini par la quantité de fibres G) chez Fagus sylvatica (Ruel et Barnoud, 1978) et sur Eucalyptus goniocalyx (Schwerin, 1958). II reste que, dans notre échantillon, le rapport $S / G$ évolue conjointement avec l'AMF et le taux de lignines de Klason, qui sont peut être les paramètres physiquement significatifs. Nos observations traduiraient alors des corrélations entre caractéristiques structurales, par exemple entre teneur globable en lignines et composition qualitative de ces lignines, sans rapport immédiat avec les DLM. Ainsi, selon le modèle de formation des parois proposé par Terashima (1990), la cinétique de lignification est telle que les unités $H$, puis $G$, sont déposées dès le départ, les unités $S$ arrivent plus tardivement. Ceci explique que la lamelle moyenne, lignifiée en premier, soit beaucoup plus riche en unités $\mathrm{G}$ que les parois secondaires. Un défaut de lignification peut être le résultat d'une cinétique de lignification particulière. II est alors associé à une composition en unités $S$ et $G$ particulière. Par exemple, une lignification tardive se traduit d'une part par une diminution de la quantité globale de lignines, d'autre part par une diminution de la quantité d'unités $G$. Un retard de lignification dans les bois à fortes DRLM pourrait donc être à l'origine des résultats constatés : taux de lignine de Klason globalement plus faible, associé à une faible lignification dans l'ensemble $\mathrm{ML}+\mathrm{P}+\mathrm{S} 1$ et à une diminution du nombre d'unités $\mathrm{G}$. 
Une piste d'étude future pourrait être la suivante : la divergence phylogénétique entre Gymnospermes et Angiospermes est dans la plupart des cas vérifiée au niveau xylologique par le fait qu'elles forment respectivement du bois de compression et du bois de tension (définition structurale). Cependant, et toujours selon une définition structurale (sans caractérisation mécanique), plusieurs auteurs ont montré l'existence de cellules analogues à du bois de tension chez des Gymnospermes et des cellules analogues à du bois de compression chez des Angiospermes (Höster et Liese, 1966 ; Jacquiot et Trénard, 1974 ; Yoshizawa et al, 1993). II serait donc utile de mieux caractériser ces bois de tension atypiques, selon leur DRLM et des marqueurs biochimiques et ultrastructuraux, et de discuter leur fonction de réorientation. Cela permettrait sans doute, en balayant des fenêtres expérimentales originales, de progresser dans l'étude des relations entre structure, biochimie et propriétés mécaniques, et éventuellement fonction de réorientation, du bois. En outre, ce type d'étude, en qualifiant la continuité des structures, peut intéresser des recherches fondamentales sur l'évolution des plantes.

\section{CONCLUSIONS ET PERSPECTIVES}

Chez les clones d'Eucalyptus hybrides du Congo, l'accroissement des DRLM s'accompagne de modifications structurales et biochimiques importantes:

- changement de la forme des cellules ;

- tendance à l'augmentation de l'épaisseur de la paroi ;

- diminution de l'angle des microfibrilles de cellulose ;

- diminution de la teneur en lignines totales (lignines de Klason) ;

- augmentation du rapport $S / G$ des unités monomériques non condensées.
Les oppositions signalées en introduction entre bois de compression et bois de tension montrent bien qu'il semble exister une variation continue des propriétés anatomiques, ultrastructurales et biochimiques entre des cellules lignifiées dont la différenciation génère des contraintes de maturation en traction ou compression longitudinale:

- bois tendu à fort retrait de maturation : diminution de la teneur en lignines, augmentation de la teneur en cellulose cristalline, diminution de l'angle des microfibrilles de cellulose, dans la couche S2 ou dans une couche supplémentaire $G$;

- bois de compression à fort gonflement de maturation : augmentation de la teneur en lignines, augmentation de l'angle des microfibrilles de cellulose dans la couche S2.

La mesure de la DRLM permet de classer quantitativement et continûment les bois, en évitant la dichotomie artificielle en bois de réaction et bois normal ou opposé. En outre, cette propriété est plus directement reliée à la fonction de réorientation du bois de réaction. On peut alors espérer progresser significativement dans la connaissance d'une part des mécanismes utilisés par les plantes pour créer des efforts internes capables de réguler ou modifier leur forme en réaction à des stimulus internes ou externes, d'autre part de la structure des tissus associés à ces fonctions de réorientation. D'une façon générale ces travaux visent à la compréhension biochimique et ultrastructurale des propriétés physicomécaniques des tissus lignifiés.

De telles études ont un intérêt technologique en mettant en évidence les conséquences de croissances et de différenciations particulières sur l'aptitude à la transformation du bois. Cela suggère alors au forestier des moyens d'action pour améliorer les potentialités de ses bois (amélioration génétique et sylvicole, tri et classement des bois abattus). 
Parmi les relations observées entre propriétés mécaniques et structure du bois, il est logique de distinguer des corrélations explicables par la seule mécanique (comme la relation entre AMF et DLM) qui risquent d'être plus stables et moins dépendantes de l'espèce et de l'échantillon, et des corrélations plus complexes traduisant des liaisons entre variables structurales, éventuellement indépendantes des propriétés mécaniques. Cette recherche requiert des modélisations micro-mécaniques réalistes et l'observation de situations biologiques très différentes ; le présent travail est un préliminaire à ce type d'étude.

\section{REMERCIEMENTS}

Nous tenons à remercier Bernard Thibaut, coordinateur du programme "Architecture, structure et mécanique de l'arbre", qui a largement participé, intellectuellement et pratiquement, à cette étude ; le personnel du CIRAD-Forêt et de l'Unité d'afforestation industrielle du Congo qui nous a permis de réaliser le prélèvement des échantillons utilisés pour cette étude ; le personnel et les chercheurs du laboratoire de paléobotanique et évolution des végétaux de l'université de Montpellier II qui ont mis à notre disposition leurs compétences et le matériel nécessaire à l'étude anatomique.

\section{RÉFÉRENCES}

Archer RR (1986) Growth Stresses and Strains in Trees. Springer Series in Wood (Timell, ed), Springer Verlag, New York, $240 p$

Archer RR (1987) On the origin of growth stresses in tress, part 1: Micromechanics of the developing cambial cell wall. Wood Sci Technol 21, 139-154

Archer RR (1988) On the origin of growth stresses in tress, part 2 : Stresses generated in a tissue of developing cells. Wood Sci Technol 23, 311-322

Baillères H, Loup C, Fournier, M Chanson B, Gérard J (1992) Contraintes de maturation en fonction de la hauteur et de l'âge chez de jeunes Eucalyptus de plantation. In : $4^{e}$ Séminaire "Architecture, Structure, Mécanique de larbre", LMGC, Université de Montpellier II, France

Baillères H (1994) Précontraintes de croissance et propriétés mécano-physiques de clones d'eucalyptus
(Pointe Noire, Congo) : hétérogénéités, corrélations et interprétations histologiques. Thèse en sciences du bois de l'université de Bordeaux I, $161 p$

Bamber RK (1987) The origin of growth stresse: A rebutal. IAWA Bulletin ns 8, 80-84

Bland DE (1958) The chemistry of reaction wood. Part I. The lignins of Eucalyptus goniocalyx and Pinus radiata. Holzforschung 12, 36-42

Bland DE (1961) The chemistry of reaction wood. Part Ill. The milled wood lignins of Eucalyptus goniocalyx and Pinus radiata. Holsforschung 15, 102-106

Bonnin A, Demanet A (1993) Micromécanique du bois durant sa formation à l'échelle de la paroi cellulaire. Mémoire de maitrise de mécanique, université de Bordeaux I, $29 \mathrm{p}$

Boyd JD (1972) Tree growth stresses. V. Evidence of an origin in differentiation and lignification. Wood SCl Technol 6, 251-262

Boyd JD (1977a) Basic cause of differentiation of tension wood and compression wood. Australian For Res 7, 121-143

Boyd JD (1977b) Relationship between fibre morphology and shrinkage of wood. Wood Sci Technol 11, 3-22

Boyd JD (1980) Relationships between fiber morphology, growth strains and physical properties of wood. Australian For Res 10, 337-360

Boyd JD (1985) The key factor in growth stress generation in trees: lignification or crystallisation. IAWA Bulletin 6, 139-150

Chanson B (1993) Déformation de maturation : héterogénéités angulaire en fonction du plan d'organisation des arbres. Acta Bot Gallica 140 (4), 395-401

Clifford MN (1974) Specificity of acid-phloroglucinol reagents. J Chromatog 94, 321-324

Delavault $O$ (1991) Anatomie dynamique et forme des arbres : cartographie du bois de tension dans de jeunes axes aériens d'Eperua falcata Aubl (Caesalpiniaceae) et essai d'interprétation. DEA «Écosystèmes forestiers tropicaux" de l'université de Montpellier II, France, $63 \mathrm{p}$

Dot P, Gautié A (1928) Manuel de technique botanique Histologie et microbiologie végétales. J Lamarre, Paris $250 \mathrm{p}$

Effland M (1977) Modified procedure to determine acid insoluble lignin in wood pulp. Tappi $60,143-144$

Fisher JB, Wassmer Stevenson J (1981) Occurrence of reaction wood in branches of dicotyledons and its role in tree architecture. Bot Gaz 140, 82-95

Fournier M (1989) Mécanique de l'arbre sur pied : maturation, poids propre, contraintes climatiques dans la tige standard. Thèse en science du bois, INP de Lorraine, Nancy, France

Fournier M, Chanson B, Thibaut B, Guitard D (1991) Mécanique de l'arbre sur pied: modélisation d'une structure en croissance soumise à des chargements permanents et évolutifs. II. Analyse tridimensionnelle des contraintes de maturation - cas du feuillu standard. Ann Sci For 48, 527-546 
Fournier M, Chanson B, Thibaut B, Guitard D (1994) Mesures des déformations résiduelles de croissance à la surface des arbres. Observations sur différentes especes. Ann Sci For 51, 249-266

Fournier M, Guitard D (1994) Les contraintes de croissance générées par la différenciation cellulaire. Acta Bot Gallica 140, 389-394

Geiger H, Fuggerer F (1979) Uber den Chemismus der Wiesner-Reaktion auf lignin. Naturforschung 34B, 1471-1472

Hejnowicz Z (1967) Some observations on the mechanism of orientation movement of woody stems. Amer $\checkmark$ Bot 54, 684-689

Höster HR, Liese W (1966) Ueber das Volkommen von Reaktiongewebe in Wurden und Asten der Dikotyledonen. Holzforschung 20, 80-90

IAWA (1964) Multilingual Glossary of Terms used in Wood Anatomy Committee on Nomenclature - International Association of Wood Anatomists (IAWA secretary, ed). Verlagsanstalt Buchdruckerei Konkordia Winterthur, Zurich, $186 \mathrm{p}$

Jacquiot J, Trénard Y (1974) Note sur la présence de trachéides à parois gélatineuses dans des bois résineux. Holzforschung 28, 74-76

Lapierre C, Monties B, Rolando C (1986a) Thioacidolysis of poplar lignins: identification of monomeric syringyl products and characterization of guaiacylsyringyl lignin fraction. Holzforschung 40, 113-118

Lapierre C, Monties B, Rolando C (1986b) Preparative thioacidolysis of spruce lignin: isolation and identification of main monomeric products. Holzforschung $40,47-50$

Lapierre C, Monties B, Rolando C (1987) Degradation of various lignins and lignin model compounds by thioacidolysis. 4th int Symp Wood Pulp Chem, Paris, Proc 2, 431-435

Laroze S, Barrau JJ (1987) Mécanique des structures. Tome 4. Calcul des structures en matériaux composites. Eyrolles-Masson, Paris, France

Lenz O, Strassler HJ (1959) Contribution à l'étude de l'éclatement des billes de hêtre (Fagus sylvatica L). Schwein Anstalt Forstl Versuchw 35, 373-409

Lewin M, Goldstein IS (1991) Wood structure and composition. "International fiber science and technology». Vol 11, Marcel Dekker Inc, New York, $488 p$

Low AJ (1964) Compression wood in conifers - A review of literature. Forestry Abstracts 25, 1-14

Mariaux A, Vitalis-Brun A (1983) Structure fine du bois de tension de Wapa en relation avec les contraintes de croissance. Bois et Forêts des Tropiques 199, 43-56

Meylan BA (1967) Measurement of microfibril angle by $X$-ray diffraction. Forest Prod J 17, 137-143

Monties B (1991) Lignins in Methods in Plant Biochemistry Vol 1 (JB Harbone, ed). Academic Press, London, 113-153

Morey PR (1973) How tree grows. Ch 4. Reaction Wood. Edward Arnold, London
Morohoshi N, Sakakibara A (1971) The chemical composition of reaction wood. Mokuzai Gakkaishi 17, 400-404

Nicholson JE, Hillis WE, Ditchburne N (1975) Some tree growth-wood properties relationship of Eucalyptus. Can J For Res 5, 424-432

Okuyama T, Yamamoto H, Masaki I, Yoshida M (1990) Generation process of growth stresses in cell walls. II. Growth stresses in tension wood. Mokuzai Gakkaishi 36, 797-803

Okuyama T, Yamamoto $H$, Yoshida M, Hattori $Y$, Archer RR (1994) Growth stresses in tension wood: role of microfibrils and lignification. IUFRO All division 5 Conference Nancy, France. Ann Sci For 51, 291-300

Okuyama T, Sugiyama K, Yamamoto H, Yoshida M (1993) Generation process of growth stresses in cell wall: relation between longitudinal realised strain and chemical composition. Wood Sci Technol 27, 257-262

Rolando C, Monties B, Lapierre C (1992) Thioacidolysis in Methods in Lignin Chemistry (SY Lin, CW Dence, eds), Springer Series in Wood Science, Springer Verlag, Berlin, Allemagne

Ruel K, Barnoud F (1978) Recherche sur la quantification du bois de tension chez le hêtre : signification statistique de la teneur en galactose. Holzforschung 32 (5), 149-156

Scurfield G (1972) Histochemistry of reaction wood cell walls in 2 species of Eucalyptus and in Tristana conferta R BR. Aust J Bot 20, 9-26

Sinnott EW (1952) Reaction wood and the regulation of tree form. Am J Bot 39, 69-78

Starkanen KV, Hergett HL (1971) In : Lignins - Occurrence, Formation, Structure and Reactions (Starkanen KV, Ludwig $\mathrm{CH}$, eds), Wiley-Interscience, New York, États-Unis, p 73

Terashima N (1990) A new mechanism for formation of a structurally ordered protolignin macromolecule in the cell wall of tree xylem. J Pulp Paper Sci 16, 150-155

Trénard Y, Guéneau P (1975) Relations entre contraintes de croissance longitudinales et bois de tension, dans le hêtre (Fagus sylvatica L). Holforschung 29, 217 223

Vigneron $P$ (1992) Création et amélioration de variétés hybrides d'Eucalyptus au Congo. Bois et Forêts des Tropiques 234, 29-43

Wilson BF, Archer RR (1977) Reaction wood: induction and mechanical action. Ann Rev Plant Physiol 28 23-43

Yamamoto H, Okuyama T (1988) Analysis of the generation process of growth stresses in cell walls. Mokuzai Gakkaishi 34, 788-793

Yoshida M, Okuyama T, Yamamoto $H$ (1992) Tree forms and internal stresses. 111. Growth stresses of branches. Mokuzai Gakkaishi 38, 663-668

Yoshizawa N, Watanabe N, Yokota S, Idei T (1993) Distribution of guaiacyl and syringyl lignins in normal and compression wood of Buxus microphylla Var insularis Nakai. IAWA Journal 4, 139-151 